\title{
Recent Advances in the Study of Inward Flow Cooling of Turbine Blade in Rotating Passages
}

\author{
Dai Ping \\ College of Electromechanical Engineering, Qingdao \\ University of Science and Technology \\ Qingdao, China \\ e-mail: daiping936@163.com
}

\author{
Bai Yang \\ College of Electromechanical Engineering, Qingdao \\ University of Science and Technology \\ Qingdao, China \\ e-mail:61496998@qq.com
}

\begin{abstract}
The development of a new generation of high performance gas turbine engines requires gas turbines to be operated at very high inlet temperatures, which are much higher than the allowable metal temperatures. Consequently, this necessitates the need for advanced cooling techniques. Among the numerous cooling technologies, the inward flow cooling technology has superior advantages and relatively favorable application prospect. The recent research progress of the inward flow cooling of turbine blade in rotating is reviewed. Influence of passage section on heat transfer in rotating and the effect of rotating smooth passage on turbulent flow and heat transfer are discussed. The influence of rotating on impinging cooling and heat transfer of rotating cooling duct with staggered pin fins is also generalized. In the last, it is noted that major problems due for further research include: optimizing of inward passage configuration and synthesis study of rotation effect on flow and heat transfer in pin-fins duct. In addition, the interactional mechanism of inward flow and film cooling will be also investigated thorough.
\end{abstract}

Keywords- gas turbines; cooling blades; conservative heat transfer; rib; rotating

\section{INTRODUCTION}

The modern gas turbine engine performance is constantly improved with the compression ratio of the engine and the turbine inlet gas temperature, and cooling structure of engine hot end components become more complex with the increase of gas temperature before the turbine. At this time, turbine blade internal cooling structure and cooling mode become one of the key technologies. Internal cooling is the flow of coolant heat transfer inside the blade passage and absorbs heat from the blade, at present the internal cooling method mainly has the impinging jet cooling, strengthening heat transfer of fin spoiler and heat transfer enhancement rib - column.

The comprehensive effect of turbulence, variation characteristics, surface roughness and channel geometry will influence characteristics of the static blade cooling fluid. Air cooled blade flow and heat of the rotating condition are far greater than the difficulty of the stationary state, because of rotational force to join compared with the stationary blade, which makes the flow analysis of cooling medium one dimension increase, rotational Coriolis force and rotating buoyancy changes the flow field, causing two flow in the channel, thus affecting the heat transfer. Therefore, the effect of rotation on the turbine internal flow field and heat transfer have a very important role for the design and optimization of gas turbine cooling in order to improve the aerodynamic performance of the turbine.

This paper summarizes the rotating condition, the research results of gas turbine blade internal cooling technology at home and abroad. In addition, the inward flow cooling should be further studied to provide reference for the rational design of turbine blade internal cooling system under rotating state in the future.

\section{EFFECT OF ROTATION ON THE FLOW FIELD}

There was a significant difference between the rotating channel flow in the channel and the static flow, centrifugal force caused by the static pressure distribution changes in flow in $U$ channel has little effect for the rotation of the turbine blade internal flow, but if the rotation number is large, the centrifugal force on the flow of the air compression effect can't be ignored. Under rotation, Coriolis force will make the flow structure has two significant changes: one is the mainstream to offset the direction of the Coriolis force. The other is a rotating radial velocity by the force caused by the Coriolis two flow, it not only changed the distribution of velocity, velocity fluctuation and random characteristics of turbulence will also change. The main direction and fluid centrifugal buoyancy of the inertial force in the same line, compared to its influence on the flow in the channel with the Coriolis force, in a weak position. Cheah et al. [1] measure rotating speed of the double channel flow using the laser Doppler velocimetry (LDV). The velocity vector diagram shows the flow separation downstream of 180 degrees elbow in Fig. 1. The reattachment length increases the forward rotation, reverse rotation so that the reattachment length decreases. Measurements show that the transverse fluctuation near the elbow matches fluctuation along the streamline direction.

\section{EFFECT OF ROTATION ON HEAT TRANSFER}

The additional force generated in the rotating turbine blade under the condition of heat transfer enhancement, flow resistance is reduced, but the rotation of the heat exchanger in each surface uneven distribution, increasing the temperature gradient. The buoyancy of centrifugal force will be derived, the near wall heat flux density 
relatively small body to accelerate the rotation axis direction, which is relatively high in the center of cold fluid density to the direction of centrifugal acceleration, causing the wall thermal fluid flow is blocked, the average thermal fluid flow rate decreased, so in the wall surface fluid and heat transfer surface of the heat exchange capacity decreased, which is an important factor that can't be ignored in high speed rotation and large temperature difference between the high pressure turbine blade.

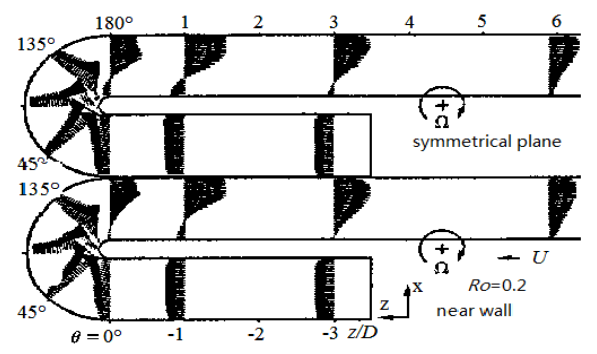

(a) Positive direction of elbow

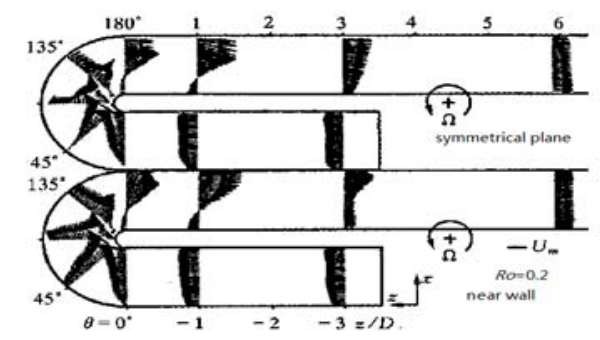

(b) Opposite direction of elbow

Figure1. The velocity distribution in the rotating U-shaped elbow

The effect of buoyancy force and the derivative Coriolis force itself has the same function, which enhances heat transfer channel pressure side, and decrease the suction side of the heat transfer effect. In the case of rotating heat transfer, mainly depends on the rotation number $\quad R_{0}=\omega D / V$ and density ratio $D R=\left(\rho_{\text {in }}-\rho_{\text {wall }}\right) / \rho_{\text {in }}$ The rotation number is the relative strength of Coriolis force and inertia force of the overall flow ratio, density ratio is the relative intensity of rotation force and inertia force of the ratio of the whole.

\section{EFFECT OF ROTATION ON THE TURBULENT FLOW FIN}

In high speed rotating conditions, convection heat transfer of rib pipe wall is a hot inner channel of convective heat transfer research in recent years. Periodic heat transfer fin is arranged on the surface of the disturbance of the boundary layer, strengthen the blade surface heat transfer, fluid separation rib caused by increased turbulence in the boundary layer, and caused by the effect of rotation speed is not as significant as the distribution of distortion in smooth channels. In addition, the fins and the whole flow into a certain angle placed in a fluid flow will cause the second flow, rotating development of two flows interaction, the fluid characteristics of fluid characteristics fin channel with smooth channels are quite different, the velocity distribution varies greatly by Han et al. [2].

In the process of rotating double square section rib in the channel, the first process and turn the front half of the velocity distribution will be affected by the direction of rotation (forward and reverse rotation) effect, but in turn affect downstream, the direction of rotation is not significant by Lacovides et al.[3]. Fig.2 shows the first and second bend channel rotation velocity distribution, the relative position of the fin, turning direction and rotation can be seen from the figure. In turn, the whole flow clockwise turn the first channel, and the overall flow to the counter clockwise turn in the second corners.
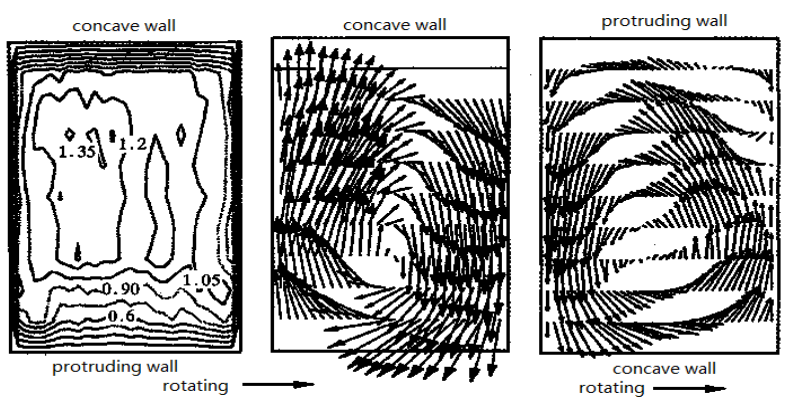

Figure 2. The second flow of passages at the elbow

\section{HEAT TRANSFER OF THE ROTATING OBLIQUE FIN CHANNEL}

The internal cooling fins in general will take different orientation (shown in Fig.3), to enhance the disturbance and increase the heat transfer area, so as to effectively reduce the temperature of the inner wall of the blade. Fin angle has a significant effect on the heat transfer distribution, the Nusselt number of 60 degrees oblique ribs than that's of 90 degrees horizontal fin produced higher, the heat transfer difference in the first and second process flow trailing edge wall is more significant.

The effect of heat transfer were compared with 60 degrees inclined rib (section is square) and 45 degrees inclined rib (section is semicircular) by Zhang et al. [4]. In the first process, Nusselt number ratio of 60 degrees oblique fin tip surface equal that's of 45 degrees oblique ribs, but the Nusselt number of 60 degrees oblique fin trailing edge surface is to be much larger than that's of 45 degrees inclined rib. In the second process, Nusselt number of the front surface 60 degrees inclined rib is relatively large, and that's of the posterior surface is relatively small. This is because in the first process, geometric features (such as the rib shape, fin orientation) on trailing edge thin boundary layer effect of leading edge effect on the large thick boundary layer. 


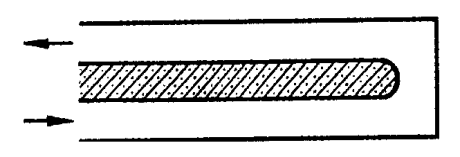

(a) Smooth no fin

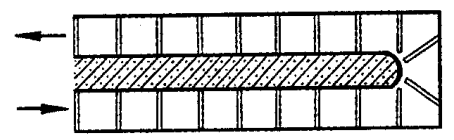

(b) 90 degrees vertical fin

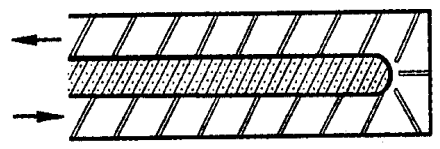

(c) 60 degrees parallel fin

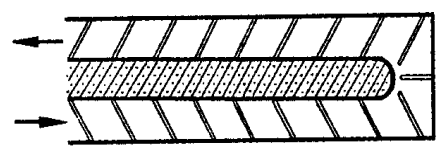

(d) 60 degrees parallel fin

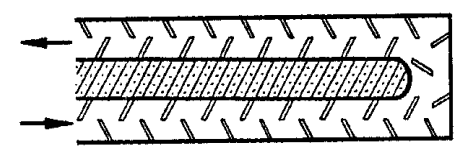

(e) 60 degrees intercross fin

Figure 3. Different fin orientation of double flow channel

\section{EFFECT OF ROATION ON THE IMPINGEMENT COOLING}

In all the technology of enhancing heat transfer, impingement cooling is the most effective method to strengthen the cooling of the local heat transfer coefficient [5-6]. By the impact of small air holes, with very high speed impact to the blade inner surface, effectively cooling on the inner surface, a portion of the exhaust leaves by gas film hole, the other part through the flow channel, and finally from the trailing edge discharge. Due to the impact of holes are arranged in the interior leaves weakened the strength of blade, so the impact is generally used in leaf blade cooling heat load is relatively large. From the fluid flow and heat transfer characteristics, the form of cooling blade leading edge is a small space for a large curvature concave flow coupled with the impact of the cooling gas in front and gill area of film hole, and the flow and heat transfer by Coriolis force, centrifugal force and non isothermal field derived buoyancy force, so blade rotating flow and heat transfer is very complex.

The impact of film cooling on leading edge of turbine blade is a typical aero engine rotating flow and heat transfer characteristics are studied by Zhu et al. [7-8] using numerical simulation method. The calculation model of orifice structure is simplified to a joint structure, and comprises an inlet block, leading edge and trailing edge block. Through the calculation of different rotation speed of the results of the analysis show that, when the Reynolds number is low before the trailing edge of the impact of the average Nusselt number distribution fluctuation phenomenon, when the Reynolds number is high, the impact of the trailing edge of the average Nusselt number distribution decreases monotonously with increasing speed, and the impact of the trailing edge of heat exchange of heat shock front surface good. In the rotation under the condition of limited space with the holes in the impact of heat transfer experiments were carried out by Xu et al. [9], and proposed a "shock / sparse film cooling structure within the confined space". The impact and rotation in the same direction and the opposite case, by changing the impact of Reynolds number, rotation number and dimensionless temperature impact on the target surface average heat transfer characteristics were studied. The study found that, the target surface heat shock increased with the increase of Reynolds number, rotation on the impact of heat transfer weakens in the Reynolds number increases obviously.

\section{THE MUTUAL INFLUENCE OF COOLING AND FILM COOLING INSIDE THE ROTATING STATE}

The film hole wall is often ribbed in order to the increase of convective heat transfer in the internal cooling passage of turbine blade in the actual. But due to the disturbance of the ribbed rib and the formation of the second flow of gas film hole flow effect, and the gas film hole layout is not single, behind the film hole of the film hole flow and the front will be different due to flow. So the internal cooling of turbine blade research, should be considered with ribs and film hole flow exist at the same time. Compared with the stationary blade, blade rotates by the Coriolis force and the centrifugal force will make the flow of internal cooling passages become very complex, change the airflow into the film holes, the changes will inevitably lead to the result of the film hole flow coefficient, influence of cooling flow so the flow coefficient and film hole rotation in effect, has very important significance in calculation and design of turbine blade cooling structure of air quantity.

At present, domestic and international research in this area is not very much. 90 degrees ribs and film hole threedimensional flow field of rotating rectangular duct flow are numerical simulated by $\mathrm{Li}$ et al. [10]. The study found that with ribs and film hole flow field in rotating inner cooling channel flow are very complex, with strong threedimensional characteristics. When the rotation number is not equal to 0 , the rotation effect has obvious influence on the discharge coefficient of film cooling hole, channel rotates clockwise, Coriolis force from the wall to bring film hole under the wall, causing the flow coefficient increases; channel counterclockwise rotation, the opposite is the case. In addition, the flow coefficient of two holes with a radial position within the channel is different, channel rotates clockwise, flow coefficient is larger than the left hole channel, counterclockwise rotation, the opposite is the case. 


\section{SUGGESTION}

The trend of research status and development of internal cooling are introduced about gas turbine blade by the rotating condition in this paper. The research work is focused on the rotational disturbed flow type fin cooling medium channel heat transfer research and rotation on the leading edge of impingement cooling effect from the development of gas turbine blade internal cooling in recent years. Through the analysis, the optimization design for turbine blade internal cooling passage should focus on the following aspects:

(1) In the case of rotating, there should be research new cooling structure, such as the structure, the rib arrangement, channel section shape, in order to further optimize the structure of flow channel and effectively protect the blade through reducing the temperature of the blade,

(2) The trailing edge region of the actual engine turbine blade is rotating the trapezoidal channel. Therefore, another focus of the future research is the effects of rotation on the trapezoidal pin fin channel flow and heat transfer.

(3) In order to optimize the cooling effect of cooling technology, composite turbine blade is widely used in cooling and film cooling inside the combination to ensure the reliability of turbine operation. Under rotating state and the external flow of film cooling internal interaction, the relationship between the two is rarely reported in the literature. The influence degree between the two effects should be further researched in order to simulate the turbine blade more real work conditions, and thus more effective cooling on the blades and ultimately improve the overall performance of the engine.

\section{REFERENCES}

[1] S. C. Cheah, H. Lacovides, and D.C. Jackson, "LDA Investigation of the flow development through rotating U-ducts,” ASME Journal of Turbomachinery, vol. 118, Dec.1996, pp. 590-595.

[2] J. E. Han , S. Dutta, and S.V. Ekkad, "Gas turbine heat transfer and cooling technology,” Xian, Xi'an Jiao Tong University press,2005.

[3] H. Lacovides, D. C. Jackson, and H. Ji, "LDA study of the flow development through an orthogonally rotating U-bend of strong curvature and rib roughened walls," ASME Journal of Turbomachinery, vol. 120, Dec. 1998, pp. 386-391.

[4] Y. M. Zhang, J. C. Han, and J. A. Parsons, "Surface heating effect on local heat transfer in a rotating two-pass square channel with 60 angled rib turbulators,” ASME Journal of Turbomachinery, vol.117, Dec. 1995, pp. 272-278.

[5] J. H. Wagner, B. V. Johnson, and R. A. Grazian, "Heat transfer in rotating serpentine passages with trips normal to the flow," ASME Journal of Turbomachinery, vol.14, Dec. 1992, pp. 847-857.

[6] H. K. Liu, Z. Tao, and S. D. Ding, "Local heat transfer in a rotating smooth and ribbed U-shaped channels,” ACTA Astronautica Sinica, vol. 27, Dec. 2006, pp. 751-755.

[7] J. R. Zhu, H. Wu, and Z, Tao, "Fluid flow and heat transfer characteristics in the lead of blades at rotating state," Journal of Beijing University of Astronautics, vol.31, Dec.2005, pp. 261-264.

[8] J. R. Zhu, and H. Wu, "Numerical study on the leading edge of turbine blade impingement and film composite cooling,"Journal of Hubei University Technology, vol. 21, Dec. 2006, pp. 50-53.

[9] L. Xu, H.P. Chang, and J. R. Pan, "Heat transfer of impingement in limited room configuration with effusion in rotating state,” Journal of Propulsion Technology, vol. 29, Dec. 2008, pp. 149-152.

[10] G. C. Li, H. R. Zhu, and T, Guo, "Effects of rotating on the discharge coefficients of film holes in turbine blade,” Journal of Propulsion Technology, vol.27, Dec. 2006, pp.394-398. 